\title{
Tratamiento de la endometritis puerperal con sulbactam-ampicilina
}

\author{
Gustavo Márquez Mariño*; Lucio Guerra Galue**
}

RESUMEN: Consideranido que la flora bacteriana sufre procesos y cambios dinámicos que modifican su respuesta a los antibióticos, obliga a que se presenten avances en antibióticoterapia.

Teniendo en cuenta que el principal problema de la unidad de sépticas es la infección obstétrica y de ella principalmente la endometritis puerperal, fue nuestro deseo poder contar con una nueva alternativa de tratamiento a los esquemas de combinación de antibióticos que el servicio ha utilizado hasta el momento obteniendo ventajas tales como cubrimiento de amplio espectro con monoterapia, continuidad de tratamiento por vía oral y baja toxicidad.

Se tomaron 22 pacientes con Dx de endometritis puerperal, y se sometieron a tratamiento con Sulbactam/Ampicilina. Se efectuaron evaluaciones clínicas diarias y pruebas de laboratorio periódicas. La Escherichia Coli y los anaerobios fueron los principales causantes de esta infección. Se obtuvo curación en el $95 \%$ de los casos y en ninguno hubo toxicidad. La estancia se redujo en un $25 \%$.

Se concluye que el antibiótico en estudio permite efectuar terapia eficaz, segura y adicionalmente reduce costos de hospitalización.

PALABRAS CLAVES: Endometritis puerperal, miometritis, sulbactam ampicilina, reducción estancia hospitalaria, eficacia.

SUMMARY: Taking account that bacterial flora present dynamic changes that modifies his response to antibiotic therapy is necessary to modify antibiotic therapy accordingly.

The basic problem of a septic unit comes from obstetric infection, mainly puerperal endometritis, we decided to evaluate the efficacy and safety of the combination sulbactam-ampicillin as a new alternative to the traditional combination of antibiotic therapy.

22 patients with puerperal endometritis were treated with the sulbactam-ampicillin combination. Clinical follow up were done on a daily basis. The more frequent bacteria isolated were Escherichia Coli and anaerobic pathogens. A cure rate of $95 \%$ was obtained. No side effects were observed. In-patient days stay were reduced in $25 \%$.

We conclude that this antibiotic is a safe and effective therapy alternative to traditional combination therapies and is cost-effective in terms of in-patient stay reduction.

KEY WORDS: Puerperal endometritis, miometritis, sulbactam- ampicillim, in patient stay reduction, efficacy.

\section{Introducción}

La endometritis es la complicación más frecuente asociada con el parto; por cesárea la incidencia varía según la población investigada entre el 5 y el $85 \%(2,8-10)$.

En nuestra institución la incidencia en cesárea está entre el 2 y el $9 \%$ mientras que en el post-parto vaginal está entre el 1 y el $2 \%$.

Los factores de riesgo más importantes para el desarrollo de la endometritis, son entre muchos, adolescencia,

\footnotetext{
* Docente adscrito Facultad de Medicina Universidad Nacional de Colombia, Jefe Unidad Sépticas Dpto. Materno Infantil Clínica San Pedro Claver ISS Bogotá. Docente de las escuelas Colombiana de Medicina, Rosario y Javeriana.

** Docente adscrito Facultad de Medicina Universidad Nacional de Colombia. Especialista Gineco-obstetra Unidad Sépticas Dpto. Materno Infantil Clínica San Pedro Claver ISS Bogotá. Instructor de las escuelas Colombiana de Medicina, Rosario y Javeriana.
}

status socioeconómico bajo, trabajo de parto prolongado y ruptura de membranas $(2,8-11)$.

Los microorganismos principalmente implicados en este tipo de infección en nuestra institución son bacilos aeróbicos gram negativos, cocos gram positivos anaerobios, bacilos gram negativos anaerobios, estreptococos del grupo B y Staphylococcus aureus $(1,2,8-10)$.

La incidencia promedio de bacteremia en pacientes con endometritis, es del $10 \%$. Menos del $2 \%$ de las pacientes infectadas desarrollan complicaciones como el shock séptico, absceso pélvico o tromboflebitis séptica $(1,2,8-10)$.

El manejo antibiótico efectivo incluye el empleo de combinación de antibióticos como Cloranfenicol/ Gentamicina o Clindamicina/Gentamicina, cefalosporinas de amplio espectro, ureido-penicilinas y últimamente el uso de una penicilina de amplio espectro, la ampicilina, asociada con un inhibidor de las betalactamasas producidas por las bacterias, el sulbactam $(1,4,6,8-10)$. 
El presente trabajo tiene como propósito comprobar la efectividad de sulbactam-ampicilina en el tratamiento de la endometritis post-parto o post-cesárea, como alternativa de tratamiento con un único antibiótico, con las ventajas que ello conlleva.

\section{Material y métodos}

Este trabajo es descriptivo, prospectivo y analítico.

A. Entre los meses febrero y marzo de 1992, se seleccionaron 22 pacientes a quienes se les hizo impresión diagnóstica de endometritis post-parto o post-cesárea, sin tener en cuenta factores de riesgo anteriores o si recibieron o no antibiótico profiláctico o terapéutico (1). Ver tabla 1.

Tabla 1

\section{CRITERIOS DIAGNOSTICOS DE ENDOMETRITIS}

Fiebre (temperatura $\geq 38^{\circ} \mathrm{C}$ )

Taquicardia (frecuencia cardíaca $\geq 90 \mathrm{p} / \mathrm{min}$.)

Sensibilidad uterina

Subinvolución uterina ( $<1-2 \mathrm{~cm}$ por día)

Loquios fétidos y/o purulentos

Leucocitosis con neutrofilia $y / o$ bandas

B. Las pacientes incluidas en el estudio fueron atendidas en su parto o cesárea en la Clímica San Pedro Claver, y evaluadas por los investigadores; una vez confirmada la impresión diagnóstica de endometritis se procedió a realizar la toma de muestras para cultivos y laboratorios, previa a la iniciación del antibiótico en estudio.

C. Se realizó la toma de muestras para Gram y cultivos bajo visión directa por especuloscopia (sin pinzamiento de cuello) directamente de cavidad uterina, utilizando escobillones de madera estériles, que se colocan en medio de transporte específico para aerobios y anaerobios y se llevaban al laboratorio para su procesamiento según las técnicas convencionales. La mayoria de las muestras se procesaron en forma inmediata y sólo algunas muestras fueron procesadas dentro de las 24 horas siguientes, período en el cual permanecieron en refrigeración. El procesamiento de las muestras fuẻ llevado a cabo siempre por el mismo laboratorista.

D. Las muestras de laboratorio que se tomaron a cada paciente se enumeran en la tabla 2. Estas mismas pruebas

Tabla 2

\section{PRUEBAS DE LABORATORIO}

Cuadro hemático completo

Tiempo parcial de tromboplastina

Tiempo de protrombina

Plaquetas

Creatinina

Nitrógeno ureico en sangre

Alanin-aminotransferasa (Alt.)

Aspartatoaminotransferasa (Ast.)

Fosfatasa alcalina

Parcial de orina fueron realizadas los días $5^{\circ}$ y $7^{\circ}$ del tratamiento para efectuar el control paraclínico del proceso séptico y evaluar la seguridad del producto (13).

E. A todas las pacientes se les inició el antibiótico en estudio en dosis de $4 \mathrm{~g}$ de ampicilina y $2 \mathrm{~g}$ de sulbactam para 24 horas divididos en 4 dosis por vía endovenosa. Ajustándonos a los parámetros internacionales mantuvimos esta dosis y vía de administración por un mínimo de 48 horas, al cabo de las cuales si los parámetros clínicos y paraclínicos mostraban persistencia o falta de resolución satisfactoria del proceso séptico, esta vía y dosis se mantenían hasta por un máximo de 10 días.

F. El seguimiento de la evolución clínica de las pacientes fue realizada diariamente por los mismos investigadores, analizando la respuesta clínica a la medicación. Ver tabla 3.

\section{Tabla 3}

PARAMETROS DE RESPUESTA CLINICA

Frecuencia cardíaca

Frecuencia respiratoria

Análisis de la curva térmica (Toma cada 4 horas)

Exploración abdominal:
A. Evaluación aspecto herida quirúrgica
B. Involución uterina
C. Sensibilidad uterina

Examen ginecológico:

A. Observación:

1. Características físicas de la secreción (Aspecto y olor)

B. Tacto vaginal:

1. Temperatura vaginal

2. Características del cuello (Consistencia y permeabilidad)

3. Evaluación integridad histerorrafía

4. Tono uterino, involución.

En caso de falta de respuesta clínica y/o paraclínica a las 48-72 horas de iniciado el antibiótico, se reevaluaba el caso y, según resultados de cultivo y antibiograma y descartando infecciones intercurrentes, se procedía al cambio del esquema antibiótico $(8,14)$.

En caso de respuesta clínica favorable, se hacía el cambio a vía oral, empleando dosis de $750 \mathrm{mg}$ de sultamicilina cada 12 horas hasta completar 7 días de tratamiento. Ver tabla 4. Algunos casos requirieron legrado uterino según criterios que se enumerarán en la tabla 5, y la paciente era dada de alta 24 horas después de iniciada esta vía, con controles cada 48 horas y toma de laboratorios en los días anteriormente indicados.

\section{Resultados y discusión}

En el presente estudio la infección post-cesárea se presentó en una proporción mayor que post-parto, comprobándose una vez más lo encontrado en la literatura universal $(1,2,8$ 10). 
Tabla 4

\section{PARAMETROS DE RESPUESTA CLINICA} FAVORABLE

1. Disminución o desaparición de los síntomas
A. Estado general
B. Dolor
C. Escalofrío
D. Fatiga Etc.

2. Desaparición de la fiebre $\left(\mathrm{T}^{\circ}\right.$ oral $\left.38^{\circ} \mathrm{C}\right)$ por más de 48 horas

3. Involución uterina

4. Aclaramiento y ausencia de fetidez en los loquios

5. FC y FR dentro de límites normales

Tabla 5

INDICACION DE LEGRADO UTERINO

1. Palpación de material endouterino sospechoso de presencia de restos.

2. Mejoría clínica favorable salvo persistencia de subinvolución uterina y/o loquios purulentos.

Es de anotar que dadas las características de nuestra institución, centro de manejo de pacientes de alto riesgo, éstas tienen factores adicionales que aumenten la infección.

Nuestras pacientes fueron mujëres jóvenes y, como era de esperarse su estatura es baja, como corresponde al pueblo colombiano. Encontramos un peso aumentado, debido a que aún no se había efectuado una completa redistribución de líquidos del embarazo (18).

El tiempo transcurrido entre el parto o la cesárea y el ingreso al servicio de sépticas fue relativamente corto aunque el rango fue amplio (3-12 días) lo que permite recalcar, una vez más, que la infección puerperal puede presentarse temprana o tardíamente dependiendo de factores como: adecuado control prenatal, estado nutricional (7), enfermedades asociadas, presencia o no de leucorreas anteparto, depresión inmunológica fisiológica del embarazo (12), etc. Asímismo; fue llamativo que nuestras pacientes consultaron oportunamente, lo que nos permitió apreciar que la severidad de los procesos no se debía a la agresividad propia de la infección obstétrica. Igualmente nos llamó la atención que las pacientes sólo recibieron droga ordenada por el médico especialista y ninguna se automedicó.

Los principales signos patológicos encontrados al examen físico inicial, fueron los relacionados con el área ginecológica.

El sistema cardíaco manifestado por taquicardia estaba comprometido en el $63 \%$ de los casos. Los demás sistemas, aunque muy importantes en sus diferentes manifestaciones (fiebre, palidez, celulitis o abscesos de herida quirúrgica, edema pretibial, etc.), porcentualmente fueron inferiores al $50 \%$ lo que permite concluir se trate de signos clínicos no frecuentes en esta entidad clínica.
De acuerdo con la literatura universal los signos clínicos que con mayor frecuencia se presentan y permiten realizar la impresión diagnóstica de endometritis se refieren a fiebre, leucocitosis y los hallazgos locales ginecológicos (1-2, 8-10, 15) que estuvieron presentes en todos los casos, con excepción de la fiebre, que en el examen físico inicial sólo se presentó en menos de la mitad de los casos, pero que durante las primeras 24 horas de hospitalización, con toma de la temperatura cada 4 horas, se comprobó que este signo clínico fue positivo en el $68.1 \%$ de los casos.

La leucocitosis fue también signo constante así como la neutrofilia y la aparición de células en banda, demostrándonos la presencia de un proceso séptico agudo. Uno de los propósitos del estudio era también comprobar si el antibiótico producía cambios a nivel de los sistemas renal, hepático o de coagulación, razón por la cual se evaluaron con los exámenes correspondientes. Lo anterior nos permitió confirmar la integridad de los sistemas al iniciar el estudio (13).

Comentario aparte merece la velocidad de sedimentación globular debido a que su elevación es fisiológica en el embarazo, lo que sólo nos permitió utilizarla como prueba de seguimiento, considerando su tendencia al aumento como una respuesta clínica inadecuada y el descenso, unida a una evolución clínica favorable como mejoría (16).

El seguimiento de la evolución clínica de nuestras pacientes fue realizado especialmente con parámetros sistémicos y locales ginecológicos.

Fue llamativo que entre 48 y 72 horas de iniciada la medicación, signos sistémicos como la taquicardia y el aumento de temperatura regresaron a cifras normales y signos locales como la fetidez de los loquios y la subinvolución uterina habían mejorado casi por completo.

Es bien sabido que el absceso de pared es una complicación de prolongada resolución dado que su proceso de formación conlleva a una serie de etapas como la celulitis, que después de su localización se presenta como absceso, momento en el cual se puede drenar pues si se intenta drenar en el período de celulitis se empeora el problema (17). Con la aplicación del antibiótico en estudio el proceso de resolución se hizo en un promedio de 6.5 días lo que nos permitió efectuar un cierre primero tardío de la herida en forma precoz y por consiguiente lograr un acortamiento en la estancia hospitalaria.

Uno de los objetivos al realizar el trabajo fue que el antibiótico a utilizar se puede emplear por vía parenteral como oral, sin tener que efectuar cambios en el medicamento o mantener la hospitalización para poder terminar el mismo. Con la droga en estudio, en promedio pudimos efectuar el cambio de vía de administración (de intravenoso a oral) al cuarto día, para completar un mínimo de 7 días de tratamiento.

El seguimiento paraclínico de los diferentes exámenes de laboratorio realizado al ingreso nos permitió comprobar, en el

Tabla 6

CEPAS RESISTENTES A SULBACTAMAMPICILINA
S. Aureus (1 de 4)
$25 \%$
S. Saprophyticus (1 de 2)
$50 \%$
P. Mirabilis (1 de 1$)$
$100 \%$ 
cuadro hemático mejoría en las cifras de hemoglobina y hematocrito como consecuencia del control del proceso séptico, mejor aporte nutricional y la redistribución completa de los líquidos corporales, disminución de las cifras leucocitarias con normalización del porcentaje de neutrófilos y linfocitos y desaparición de las células en banda, además de una marcada disminución de la VSG, coincidiendo con la mejoría clínica del cuadro séptico.

Los valores de las pruebas de coagulación, hepáticas y renales no sufrieron modificación lo que nos permitió concluir que la droga carece de efectos tóxicos sobre estos sistemas. Ver tabla 7.

Tabla 7

\section{INTERVALOS DE CONFIANZA PARA LAS DIFERENCIAS EN LOS PROMEDIOS DE EXAMENES DEL SEGUIMIENTO PARACLINICO}

\begin{tabular}{|l|c|c|c|}
\hline & $\begin{array}{c}\text { PRE } \\
\text { TRATAMIENTO }\end{array}$ & $\begin{array}{c}\text { POST } \\
\text { TRATAMIENTO }\end{array}$ & $\begin{array}{c}\text { INTERVALOS DE } \\
\text { CONFIANZA PARA LA } \\
\text { DIFERENCIA }\end{array}$ \\
\hline HEMATOCRITO & 34.59 & 35.76 & $-5.086,-2.77$ \\
\hline HEMOGLOBINA & 11.27 & 12.50 & $-1.840,0.601$ \\
\hline LEUCOCITOS & 13450 & 10030 & 2056,4783 \\
\hline NEUTROFILOS & 78.47 & 9191.611 & $6.850,15.81$ \\
\hline BANDA & 2.33 & 0 & $-16.33,8.43$ \\
\hline LINFOCITOS & 15.91 & 28.24 & $-5.485,-09768$ \\
\hline MONOCITOS & 2.54 & 5.77 & $-322180,13040$ \\
\hline PLAQUETAS & 411214 & 637500 & $0.39,16$ \\
\hline $\begin{array}{l}\text { VELOCIDAD } \\
\text { SEDIMENTACIO }\end{array}$ & 50.56 & 41.89 & $-4.62,2.54$ \\
\hline $\begin{array}{l}\text { COAGULACION } \\
\text { KPTT } \\
\text { (DIFERENCIA } \\
\text { CONTROL) }\end{array}$ & -1.24 & -0.2 & $-1.41,2.52$ \\
\hline $\begin{array}{l}\text { PROTOMBINA } \\
\text { (DIFERENCIA } \\
\text { CONTROL) }\end{array}$ & 0.16 & 0.695 & $1.145,2.52$ \\
\hline $\begin{array}{l}\text { FOSFATASA } \\
\text { ALCALINA }\end{array}$ & 140.67 & 13.83 & $-15.31,15.96$ \\
\hline ALT & 31.36 & 31.04 & 1075 \\
\hline AST & 27.43 & 22.20 & -0.29, \\
\hline
\end{tabular}

INTERVALOS A UN NIVEL DE CONFIANZA DEL 95\%

No es posible hacer la presentación de un trabajo científico sobre infección sin mostrar los resultados de los cultivos para gérmenes aerobios y anaerobios. Sin embargo, se debe aclarar que el cultivar gérmenes anaerobios es un proceso más complicado que el de aerobios, aún en laboratorios plenamente especializados para tal fin, lo que nos permite explicar al presentar nuestros resultados el porque de la diferencia entre el número de cultivos positivos para aerobios (73\%) frente al número de cultivos positivos para anaerobios (34\%) (19-20).

De las 22 pacientes estudiadas, sólo a una no se le pudo tomar muestras debido a que fue necesario intervenirla quirúrgicamente de urgencia por sangrado masivo por miometritis. A las 21 restantes se les tomaron muestras endometriales para aerobios y anaerobios y, de $\operatorname{los} 8$ casos que presentaron abscesos de pared, igualmente se les cultivó material obtenido del fondo del absceso para aerobios y anaerobios, lo que dio un gran total de 52 muestras, de las cuales sólo en 28 (53\%) se obtuvo crecimiento de gérmenes. De estas muestras, en $19(67 \%)$ crecieron gérmenes aerobios y en $9(33 \%)$ anaerobios. Ver tablas 8 y 9.

Los resultados obtenidos una vez más nos confirman el gran problema que genera la Escherichia Coli en la infección

Tabla 8

RESULTADO DE CULTIVOS Y ASOCIACIONES BACTERIANAS

\begin{tabular}{|c|c|c|c|}
\hline CASO No & AEROBIOS & ANAEROBIOS & ASOCIADOS \\
\hline - 1 & $\begin{array}{r}\text { Pseudomonas cepacia } \\
\text { (E) }\end{array}$ & $\begin{array}{c}\text { Bacteriodes spp (E) } \\
\text { Peptococcus SPP } \\
\text { (E) }\end{array}$ & SI \\
\hline 2 & $\begin{array}{|cc|}\text { ENTEROCOCOS } & (\mathrm{E}) \\
\text { Staphyloccoccus } & (\mathrm{A}) \\
\end{array}$ & 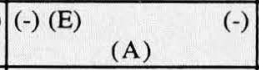 & NO \\
\hline 3 & Escherichia Coli (E) & $(-)(E)$ & NO \\
\hline 4 & $\begin{array}{l}\text { Staphylococcus (E) } \\
\text { saprophyticus }\end{array}$ & $(-)(E)$ & NO \\
\hline 5 & $\begin{array}{c}\text { Klebsiella } \\
\text { pneumoniae }\end{array}$ & Pectococcus spp (E) & SI \\
\hline 6 & Escherichia Coli (E) & $(-)(E)$ & NO \\
\hline 7 & $(-)(E)$ & $(-)(E)$ & NO \\
\hline 8 & Escherichia Coli (E) & Peptococcus spp (E) & SI \\
\hline 9 & Escherichia Coli (E) & $(-)(E)$ & NO \\
\hline 10 & $(-)(E)$ & $(-)(E)$ & NO \\
\hline 11 & NO SE I & HICIERON CULTIVC & \\
\hline 12 & $(-)(E)$ & $\begin{array}{c}\text { Peptococcuss } \text { spp } \\
\text { (E) }\end{array}$ & NO \\
\hline 13 & \begin{tabular}{|c|}
\multicolumn{2}{|c|}{ Staphylococcus } \\
Saprophyticus (A) \\
Escherichia Coli \\
Proteus mirabilis \\
\end{tabular} & $\begin{array}{c}\text { Bacteroides spp (E) } \\
\text { Bacteroides (A) }\end{array}$ & NO \\
\hline 14 & Escherichia Coli (E) & Peptococcus spp (E) & SI \\
\hline 15 & $\begin{array}{c}\text { Corynebacterium spp } \\
\text { (E) }\end{array}$ & Bacteriodes spp (E) & SI \\
\hline 16 & $(-)(E)$ & $(-)(E)$ & NO \\
\hline 17 & $\begin{array}{l}\text { Staphylococcus } \\
\text { aureus (A) Y (E) }\end{array}$ & $(-)(A)$ y $(B)$ & NO \\
\hline 18 & $(-)(E)$ & $(-)(E)$ & NO \\
\hline 19 & $(-)(E)$ & $(-)(E)$ & NO \\
\hline 20 & \begin{tabular}{|c|}
$\begin{array}{c}\text { Corynebacterium spp } \\
\text { (E) Staphylococcus } \\
\text { aeureus (A) }\end{array}$ \\
\end{tabular} & $\begin{array}{ll}(-) & (\mathrm{E}) \\
(-) & (A)\end{array}$ & \\
\hline 21 & Escherichia Coli (E) & $\begin{array}{ll}(-) & (\mathrm{E}) \\
(-) & (A) \\
\end{array}$ & NO \\
\hline 22 & Escherichia Coli (E) & $(-)(E)$ & No \\
\hline
\end{tabular}

E: ENDOMETRIO

A: ABSCESO DE PARED

ginecológica, así como el Staphylococcus aureus a nivel del endometrio $(1-2,8-11,14-15)$. En las tablas 10 y 11 se presentan los diversos microorganismos gram $(+)$ y gram () encontrados así como su porcentaje. En las muestras provenientes de absceso de pared se observó la predominancia de gérmenes gram $(+)(14,17)$. 


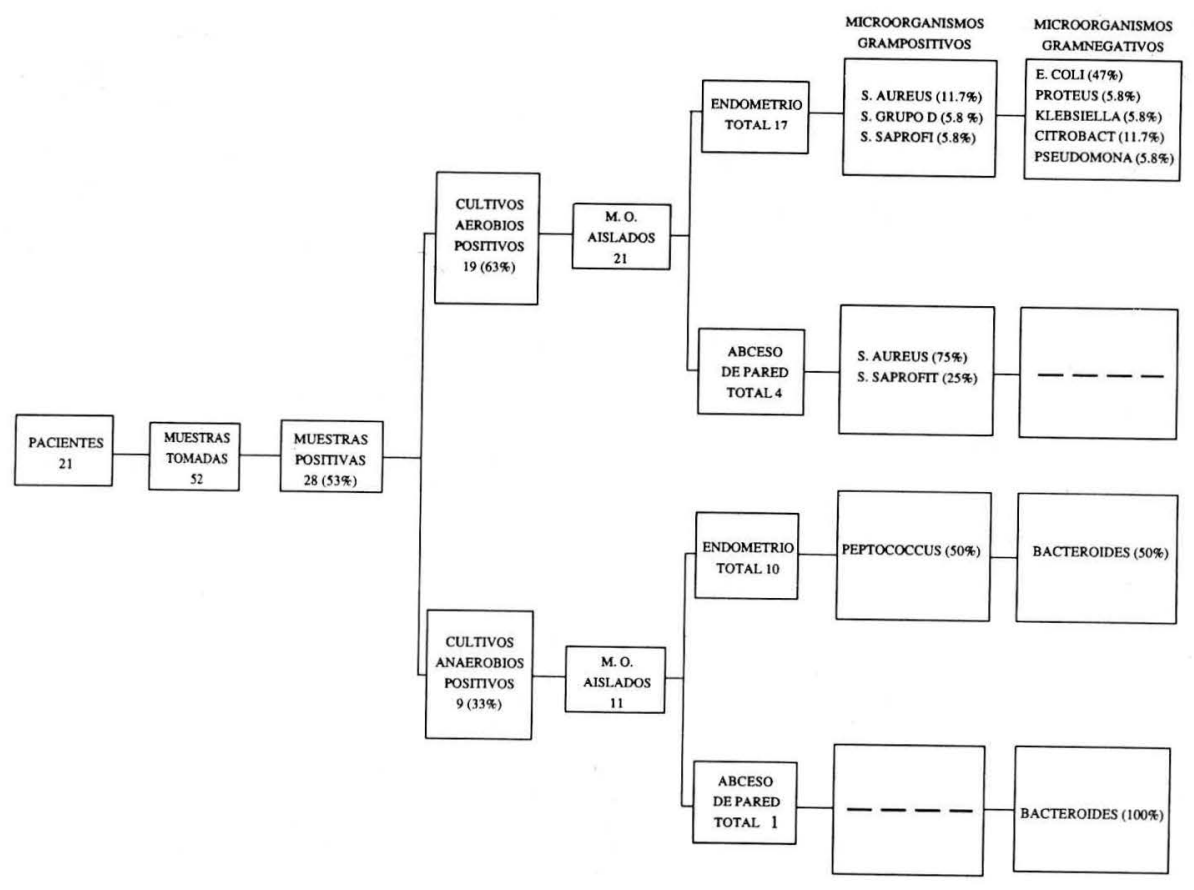

De las muestras procedentes de endometrio fue sorprendente la diversidad de microorganismos aerobios aislados, 21 en total, demostrándose una vez más que las infecciones ginecológicas son polimicrobianas debido a que su origen es la flora de vagina y cuello uterino $(1,14)$. Con relación a los cultivos de anaerobios se aislaron 11 microorganismos, los cuales en un $33 \%$ estaban asociados a aerobios y un $25 \%$ entre ellos mismos. Así como lo refiere la literatura universal, encontramos a los bacteroides y los peptococos como los gérmenes anaerobios más frecuentes causantes de infección ginecológica (1-2, 8-10, 14-15). .

En relación a los microorganismos procedentes de los abscesos de pared y gérmenes anaerobios se encontró que sólo en un caso se cultivó un bacteroides, esto es probablemente debido a que los abscesos de pared llegaron parcialmente drenados, lo que redujo aún más la positividad de la muestra para estos gérmenes. Tablas 8 y 9 .

Es importante advertir que, con base en estudios realizados por el servicio de epidemiología de la Clínica San Pedro Claver, la ampicilina fue retirada del armamento antibiótico debido a que más del $60 \%$ de los gérmenes aislados eran resistentes a este medicamento. Sin embargo, al evaluar la asociación del inhibidor de betalactamasas, sulbactam, con la ampicilina, obtuvimos una sensibilidad, en cuanto a gérmenes gram (+) muy similar al de las cefalosporinas de primera generación y para gram (-), comparable a la de los aminoglucósidos. Adicional a lo anterior se comprobó clínicamente el cubrimiento de los anaerobios $(3-5,13)$. Ver tablas 10 y 11 .
Tabla 10

SENSIBILIDAD FRENTE A LOS ANTIBIOTICOS MICROORGANISMOS GRAM POSITIVO AEROBIOS

\begin{tabular}{|c|c|c|c|c|c|c|}
\hline \multirow{2}{*}{ ANTIBIOTICO } & \multicolumn{2}{|c|}{ S. AUREUS } & \multicolumn{2}{|c|}{ S. GRUPO D } & \multicolumn{2}{|c|}{ S. } \\
\hline \multirow{2}{*}{ OXAPROPHYTICUS } \\
\cline { 2 - 7 } & No & $\%$ & No & $\%$ & No & $\%$ \\
\cline { 2 - 7 } & 3 & 75 & 0 & 0 & 2 & 100 \\
\hline CEFAZOLINA & 3 & 75 & 1 & 100 & 2 & 100 \\
\hline CEFALOTINA & 1 & 25 & 1 & 100 & 2 & 100 \\
\hline CEFUROXIMA & 3 & 75 & 1 & 100 & 2 & 100 \\
\hline CLORANFENICOL & 3 & 75 & 1 & 100 & 2 & 100 \\
\hline CLINDAMICINA & 3 & 75 & 1 & 100 & 2 & 100 \\
\hline PENICILINA & 2 & 50 & 1 & 100 & 0 & 0 \\
\hline ERITROMICINA & 3 & 75 & 1 & 100 & 2 & 100 \\
\hline TRIMET/SULFA & 4 & 100 & 1 & 100 & 1 & 50 \\
\hline VANCOMICINA & 4 & 100 & 1 & 100 & 2 & 100 \\
\hline GENTAMICINA & 3 & 75 & 0 & 0 & 1 & 50 \\
\hline TETRACICLINA & 2 & 50 & 0 & 0 & 0 & 0 \\
\hline $\begin{array}{c}\text { SULBACTAM- } \\
\text { AMPICILINA }\end{array}$ & 3 & 75 & 1 & 100 & 1 & 50 \\
\hline $\begin{array}{c}\text { TOTAL CEPAS } \\
\text { AISLADAS }\end{array}$ & & 4 & & 1 & & 2 \\
\hline
\end{tabular}


Sin embargo, observamos resistencia in vitro en 1 de los 4 Staphylococcus aureus aislados, en 1 de los 2 Staphylococcus saprophyticus aislados y en un único proteus mirabilis aislado. Ver tabla 6.

De aclararse que, salvo en el caso de la paciente en la que se le aisló el proteus mirabilis, en ninguna otra fue necesario hacer cambio de la medicación, ya que la mejoría clínica así nos lo indicó.

Debemos reiterar que, como en todo proceso séptico la terapia antibiótica debe ser complementada no sólo con las medidas generales sino con otros medicamentos que coadyuven con el objetivo terapéutico. En el caso de la ocitocina, la cual se utilizó en un promedio de $20 \mathrm{U} /$ día durante 4 días para lograr la involución uterina adecuada.

\section{Agradecimientos}

Especialmente a los doctores Gabriel Onofre y Daniel Lobo Guerrero, directivos de Laboratorios Pfizer por su extraordinaria colaboración en la realización del trabajo así como a la Bioestadística Clara Inés Martínez.
Tabla 11

SENSIBILIDAD FRENTE A LOS ANTIBIOTICOS MICROORGANISMOS GRAM NEGATIVOS AEROBIOS

\begin{tabular}{|c|c|c|c|c|c|c|c|c|}
\hline \multirow{2}{*}{ ANTIBIOTICO } & \multicolumn{2}{|c|}{ E. COLI } & \multicolumn{2}{|c|}{ P. } & \multicolumn{3}{|c|}{ K. } & \multicolumn{2}{|c|}{ P. } \\
\cline { 2 - 10 } & $\#$ & $\%$ & $\#$ & $\%$ & $\#$ & $\%$ & $\#$ & $\%$ \\
\hline AMIKACINA & 8 & 100 & 1 & 100 & 1 & 100 & 1 & 100 \\
\hline GENTAMICINA & 8 & 100 & 1 & 100 & 1 & 100 & 1 & 100 \\
\hline CLORANFENICOL & 7 & 87.5 & 1 & 100 & 1 & 100 & 1 & 100 \\
\hline TETRACICLINA & 7 & 87.5 & 1 & 0 & 1 & 100 & 1 & 100 \\
\hline CEFAZOLINA & 7 & 87.5 & 1 & 0 & 1 & 100 & 1 & 100 \\
\hline CEFALOTINA & 7 & 87.5 & 1 & 0 & 1 & 100 & 1 & 100 \\
\hline CEFOXITIN & 7 & 87.5 & 1 & 0 & 1 & 100 & 1 & 100 \\
\hline CEFOPERAZONA & 7 & 87.5 & 1 & 100 & 1 & 100 & 1 & 100 \\
\hline CEFOTAXIMA & 8 & 100 & 1 & 100 & 1 & 100 & 1 & 100 \\
\hline CEFTAZIDIMA & 8 & 100 & 1 & 100 & 1 & 100 & 1 & 100 \\
\hline CEFTIZOXIMA & 7 & 87.5 & 1 & 100 & 1 & 100 & 1 & 100 \\
\hline CEFAMANDOL & 8 & 87.5 & 1 & 100 & 1 & 100 & 0 & 0 \\
\hline CEFONICID & 8 & 100 & 1 & 100 & 1 & 100 & 0 & 0 \\
\hline CEFUROXIMA & 8 & 100 & 1 & 100 & 1 & 100 & 1 & 100 \\
\hline CEFTRIAXONA & 8 & 100 & 1 & 100 & 1 & 100 & 1 & 100 \\
\hline TRIMT/SULFA & 7 & 87.5 & 1 & 100 & 1 & 100 & 0 & 0 \\
\hline SULBACTAM/AM & 8 & 100 & 1 & 0 & 1 & 100 & 1 & 100 \\
\hline TOTAL CEPAS & & 8 & & 1 & & 1 & & 1 \\
\hline AISLADAS & & & & & & & & \\
\hline
\end{tabular}

\section{BIBLIOGRAFIA}

1. Reese R., Betts R. A practical approach to infectious diseases. MSD Handbook 3rd. Edition 1991; 428-430.

2. Faro S. Relación de las enfermedades infecciosas con la cesárea. Temas actuales Ginecología y Obstetricia 1988; (4): 739-762.

3. Quintero G., Escallón J., Ariza A. Profilaxis antibiótica con Sulbactam/ Ampicilina en pacientes que van a apendicectomía o colecistectomía. ACM: Anuario 1991; 49-55.

4. Newton E., Gibbs R. Treatment of post partum endometritis: A comparison of Ampicilina/Sulbactam vs Gentamicin plus Clindamycin. Presented at a scientific exhibit at the thirty sixth Annual Clinical Meeting of the American college of obstetricians and gynecologist. Boston, Ma, 1988.

5. Krohn K. Effect of profhylactic administration of Sulbactam/Ampicilin on the rate of postoperati endometritis after first trimester abortion. Reviews of infectious diseases. 1986; 8: 576-578.

6. Arredondo GJL. y col. Tratamiento de la endometritis post-cesárea y post-parto. Comparación de dos esquemas: Pefloxacina vs Ampicilina Gentamicina. Ginecol. Obstet. Méx. 1990; 58: 87-93.

7. Shizgal H., Nutrición y la respuesta inmune. Infección y respuesta inmune alterada en pacientes quirúrgicos. Curso Postgrado. American College of Surgeons. Eighth annual Sprin Meeting American College of Sugeons. 1980; 79-84.

8. Duff P. Pathophysiology and management of post-cesarean endomyometritis. Obstetrics and Gynecology. 1986; (67): 269-276.

9. Cox SM., Gilltrap L. Endometritis post-parto. Temas actuales Ginecología y Obstetricia. $1989 ; 2: 351-360$.
10. Sweet R., Gibbs R. Postpartum infections. Infectious diseases of the female genital tract. Parte 3. 1985; cap. 17: 277-292.

11. Monif GR. Enfermedades infecciosas en Obstetricia y Ginecología. Ed. Salvat. 1985; 523-549.

12. Feinber B., Gonik B. Conceptos generales de inmunología durante el embarazo. Clínicas Obstétricas y Ginecológicas 1991; 1: 3-16.

13. Mandell, Douglas, Bennett. Enfermedades infecciosas principios y práctica. 3a. Edición. Ed. Panamericana 1985; 20: 271.

14. Gardner P., Provine H. Manual of acute bacterial infections. Early diagnosis and treatment. 1a. Edición. Little, Brown and Company. 1985; cap. 3.

15. Pritchard J., MacDonald P., Gant NW. Obstetricia 3a. Edición. Ed. Salvat. 1986; 697-705.

16. Ballcells A. La Clínica y el Laboratorio. 15a. Edición. Ed. Salvat. 1989; Cap. 3. 132.

17. Flint L. Early postoperative acute abdominal complications. Surgical Clinics of North America. 1988; 2: 445-455.

18. Pritchard J., MacDonald P., Gant NW. Obstetricia 3a. Edición. Ed. Salvat. 1986; 355-362.

19. Ballcells A. La Clínica y el Laboratorio. 15a. Edición. Ed. Salvat. 1986; 549.

20. Gardner P., Provine H. Manual of acute bacterial infections. Early diagnosis and treatment. 1a. Edición. Little, Brown and Company. 1985; 197-200. 